\title{
Electrical Stimulation of Denervated Rat Skeletal Muscle Retards Capillary and Muscle Loss in Early Stages of Disuse Atrophy
}

\author{
Kouki Nakagawa, ${ }^{1}$ Hiroyuki Tamaki, ${ }^{1}$ Keishi Hayao, ${ }^{1}$ Kengo Yotani, ${ }^{2}$ Futoshi Ogita, \\ Noriaki Yamamoto, ${ }^{1,3}$ and Hideaki Onishi ${ }^{1}$ \\ ${ }^{1}$ Institute for Human Movement and Medical Sciences, Niigata University of Health and Welfare, Niigata, Japan \\ ${ }^{2}$ National Institute of Fitness and Sports in Kanoya, Kanoya, Japan \\ ${ }^{3}$ Niigata Rehabilitation Hospital, Niigata, Japan \\ Correspondence should be addressed to Hiroyuki Tamaki; hiroyuki-tamaki@nuhw.ac.jp
}

Received 8 January 2017; Accepted 29 March 2017; Published 13 April 2017

Academic Editor: Leonardo F. Ferreira

Copyright (C) 2017 Kouki Nakagawa et al. This is an open access article distributed under the Creative Commons Attribution License, which permits unrestricted use, distribution, and reproduction in any medium, provided the original work is properly cited.

\begin{abstract}
The purpose of the present study is to investigate the effects of low-frequency electrical muscle stimulation (ES) on the decrease in muscle mass, fiber size, capillary supply, and matrix metalloproteinase (MMP) immunoreactivity in the early stages of denervationinduced limb disuse. Direct ES was performed on the tibialis anterior muscle following denervation in seven-week-old male rats. The rats were divided into the following groups: control (CON), denervation (DN), and denervation with direct ES (DN + ES). Direct ES was performed at an intensity of $16 \mathrm{~mA}$ and a frequency of $10 \mathrm{~Hz}$ for $30 \mathrm{~min}$ per day, six days a week, for one week. We performed immunohistochemical staining to determine the expression of dystrophin, CD34, and MMP-2 in transverse sections of TA muscles. The weight, myofiber cross-sectional area (FCSA), and capillary-to-fiber (C/F) ratio of the tibialis anterior (TA) muscle were significantly reduced in the DN group compared to the control and DN + ES groups. The MMP-2 positive area was significantly greater in DN and DN + ES groups compared to the control group. These findings suggest beneficial effects of direct ES in reducing muscle atrophy and capillary regression without increasing MMP-2 immunoreactivity in the early stages of DN-induced muscle disuse in rat hind limbs.
\end{abstract}

\section{Introduction}

Limb disuse due to denervation (DN) causes musculoskeletal atrophy accompanied by a reduction in muscle tissue capillary supply. In particular, steep decreases in muscle mass and force generated by the muscle are observed in earlystage disuse atrophy. Significant decreases in muscle mass and fiber size occur within one week of denervation, and by two weeks the tibialis anterior (TA) muscle atrophies to about $50 \%$ of the control [1-3]. Muscle fiber atrophy is accompanied by increased capillary density, reduced capillary-tofiber $(\mathrm{C} / \mathrm{F})$ ratio, and decreased capillary diameter after hind limb unloading and denervation [4-6]. Capillaries supply muscle fibers with oxygen and nutrients and remove waste metabolic products. Capillary density is also reduced because less blood flow is required to meet the metabolic demands in the atrophic muscle. Therefore, therapeutic approaches that enhance blood flow induced by muscle contractions might ameliorate muscle atrophy and capillary loss during the early stages of $\mathrm{DN}$-induced disuse.

Electrical muscle stimulation (ES) has been utilized as a therapeutic intervention and a functional substitute for voluntary muscle contraction in patients with spinal cord injury (SCI) [7] and long-standing denervation. Some clinical studies have found that direct ES to denervated muscles increases muscle mass and average fiber diameter [8,9]. Moreover, the early application of ES treatment following orthopedic surgery may effectively attenuate loss of muscle strength and improve functional performance [10]. In contrast, other preclinical studies have demonstrated no beneficial effects of chronaxie-based ES (frequency: $20 \mathrm{~Hz}$; pulse duration: twice the chronaxie value; $3 \mathrm{~s}$ on, $6 \mathrm{~s}$ off) on nerve crush-induced muscle fiber atrophy and muscle excitability [11]. In fact, chronaxie-based ES has been reported 
to induce muscle fibrosis and atrophy in denervated muscles [12]. Furthermore, although high-intensity (16 mA) ES was effective in retarding denervated muscle atrophy, it may have adverse effects on the regeneration of nerve terminals in the neuromuscular junction and/or the membrane systems involved in excitation-contraction coupling [13]. The effects of ES on muscle atrophy appear to be influenced by the type of disuse model used, as well as the nature of the experimental ES regimen, which can vary in the intensity, frequency, and number of contractions [14-16].

In recent years, it has been reported that matrix metalloproteinases (MMPs) play an important role in the homeostasis and maintenance of myofiber functional integrity; MMPs break down the extracellular matrix (ECM) and regulate skeletal muscle cell migration, differentiation, and regeneration [17]. MMPs are also essential for the angiogenic response to physical activity, which is a complex process thought to be regulated by a balance of proangiogenic and antiangiogenic factors [18]. At the initial stage of capillary sprout formation, the continuous basement membrane, which is composed of type IV collagen and laminin-rich connective tissue membrane underlying endothelial cell layers, must first be dissolved by proteinases before the migration and proliferation of distinct endothelial cell populations occur. MMP-2 (66 kDa, type IV collagenase, gelatinase A) activity is important in the early stages of endothelial cell morphogenesis/capillary formation, as MMP-2 and MMP-9 are responsible for the fragmentation of basement membrane type IV collagen $9[19,20]$. Furthermore, based on inhibition studies of MMP-2, ablation of MMP-2 may impair not only angiogenesis, but also the growth of regenerated muscle fibers via decreased VEGF and nNOS expression [21]. Therefore, MMP-2 may also play a significant role in myogenesis and muscle regeneration $[22,23]$.

The activity of MMP-2 has been reported to increase following DN and limb immobilization [24, 25] or muscle stretching in the denervated muscle [26]. Similarly, chronic ES and exercise training elevate MMP-2 expression to various levels depending on intensity $[27,28]$. On the other hand, other studies show that ES treatment $(20 \mathrm{~Hz}$ for $3 \mathrm{~s}$ followed by $6 \mathrm{~s}$ of rest, repeated for 20 maximal contractions, applied every $48 \mathrm{~h}$ for 28 days) does not alter MMP-2 activity [25], indicating that discrepancies might occur among interventions with different conditions. To our knowledge, the histomorphological effects of low-frequency ES on muscle fibers, capillaries, and MMP-2 immunoreactivity in early-stage DNinduced hind limb disuse have yet to be studied, although some studies have demonstrated that even low-frequency ES (10 Hz, incomplete tetanus) reduces DN-induced muscle loss $[13,16]$. Thus, it is important to explore the potential use of low-frequency ES (e.g., $10 \mathrm{~Hz}$ ) to maintain both muscle mass and capillaries. We hypothesize that low-frequency ES reduces muscle atrophy and capillary loss in early stages of DN-induced limb disuse.

The purpose of the present study is to investigate the histomorphometric effects of low-frequency ES on the decrease in muscle mass, fiber size, capillary density, and MMP immunoreactivity in the early stages of DN-induced limb disuse.

\section{Materials and Methods}

Twenty-two male Fischer 344 rats (CLEA, Tokyo, Japan) were individually housed in standard cages at constant temperature $\left(23 \pm 2^{\circ} \mathrm{C}\right)$, humidity $(55 \% \pm 5 \%)$, and $12 \mathrm{~h}$ light-dark cycles. Rats were provided with CE-2 rodent chow (CLEA) and water ad libitum. Seven-week-old rats were randomly assigned to one of the following groups: age-matched controls (CON, $n=8)$, denervation with direct ES (DN + ES, $n=$ $8)$, or denervation without $\mathrm{ES}(\mathrm{DN}, n=6)$. The sample size ( $n=6-7 /$ group) was calculated with reference to FCSA data from previous studies from our laboratory, using the following formula $[6,29-31]$ :

$$
n=\frac{(r+1)}{r} \times \frac{\sigma^{2}\left(Z_{\alpha / 2}+Z_{\beta}\right)^{2}}{\Delta^{2}},
$$

where $r$ is the ratio of the larger group to the smaller group, $\sigma$ is the standard deviation, $\Delta$ is the effect size, $\alpha=0.05, \beta=$ 0.2 (for $80 \%$ power with $95 \%$ confidence), $Z_{\alpha / 2}=1.96$, and $Z_{\beta}=0.84$.

All animal manipulations and protocols were carried out in accordance with the guidelines presented in the Guiding Principles for the Care and Use of Animals in the Field of Physiological Sciences, published by the Physiological Society of Japan. All procedures were approved by the Animal Committee of the National Institute of Fitness and Sports and the Animal Committee of Niigata University of Health and Welfare.

The rats in the DN and DN + ES groups were anesthetized with an intraperitoneal injection $(50 \mathrm{mg} / \mathrm{kg})$ of sodium pentobarbital. An incision was made on the skin covering the left buttock and the sciatic nerve was exposed and separated from the surrounding tissue. The sciatic nerve was frozen for $5 \mathrm{~s}$ using a $5 \mathrm{~mm}$ diameter stainless steel rod cooled in liquid nitrogen $[13,16,32-35]$. This freezing procedure uniformly damages nerve fibers, similar to nerve crushing, cutting, or transection with a suture [13, 32, 34, 35]. Evoked electromyography was performed with ES at a location proximal to the freezing site of the sciatic nerve to confirm the efficacy of TA muscle denervation [36].

On the day after surgery, the left TA muscles of the DN + ES rats under isoflurane inhalation anesthesia (2\%) were percutaneously electrically stimulated. The stimulation protocol was delivered as previously described $[16,37]$. Bipolar silver surface electrodes ( $3 \mathrm{~mm}$ diameter) were briefly attached to the shaved anterior surface of the left leg. ES was performed using electrostimulators with an isolator (SEM-4201, Nihon Kohden, Tokyo, Japan) for 30 minutes a day, six days a week, for one week at an intensity of $16 \mathrm{~mA}$, a frequency of $10 \mathrm{~Hz}$, and a pulse width of $250 \mu \mathrm{s}$. The ES regimen comprised $2 \mathrm{~s}$ of stimulation followed by $6 \mathrm{~s}$ of rest. Muscle contractions induced by ES at $10 \mathrm{~Hz}$ are characterized by minimal summation during twitch contraction [16]. The ES regimen used in the previous study ( $10 \mathrm{~Hz}$ for $2 \mathrm{~s}$ followed by $6 \mathrm{~s}$ of rest, repeated for 225 cycles over $30 \mathrm{~min}$ ) caused as many as 4500 instances $(10 \times 2 \times 225)$ of muscle contraction, mechanical stimuli, or both. One week after denervation, rats in the control, DN, and DN + ES groups were anesthetized with 
TABLE 1: Body weight (BW), muscle weight relative to BW, myofiber cross-sectional area (FCSA), capillary-to-fiber (C/F) ratio, capillary density (CD), and MMP-2 positive area in the TA muscles.

\begin{tabular}{lccc}
\hline & CON & DN & DN + ES \\
\hline BW $(\mathrm{g})$ & $177 \pm 24$ & $160 \pm 21$ & $160 \pm 17$ \\
TA muscle weight $(\mathrm{mg} / \mathrm{g}$ BW) & $1.91 \pm 0.18$ & $1.23 \pm 0.04^{* *}$ & $1.62 \pm 0.21^{\dagger \dagger}$ \\
EDL muscle weight $(\mathrm{mg} / \mathrm{g} \mathrm{BW})$ & $0.48 \pm 0.08$ & $0.38 \pm 0.03^{* *}$ & $0.38 \pm 0.02^{*}$ \\
Sol muscle weight $(\mathrm{mg} / \mathrm{g} \mathrm{BW})$ & $0.37 \pm 0.07$ & $0.25 \pm 0.04^{*}$ & $0.27 \pm 0.02^{*}$ \\
TA FCSA $\left(\mu \mathrm{m}^{2}\right)$ & $1425 \pm 272$ & $952 \pm 144^{*}$ & $1365 \pm 341^{\dagger}$ \\
C/F ratio & $1.22 \pm 0.31$ & $0.73 \pm 0.19^{*}$ & $1.17 \pm 0.24^{\dagger}$ \\
CD $\left(\# / \mathrm{mm}^{2}\right)$ & $698 \pm 272$ & $620 \pm 211$ & $714 \pm 265$ \\
MMP-2 positive area $\left(\mu \mathrm{m}^{2}\right)$ & $275 \pm 148$ & $824 \pm 341^{*}$ & $1101 \pm 588^{*}$ \\
\hline
\end{tabular}

${ }^{*} P<0.05$ and ${ }^{* *} P<0.01$ versus $C O N ;{ }^{\dagger} P<0.05$ and ${ }^{\dagger \dagger} P<0.01$ versus DN.

sodium pentobarbital ( $50 \mathrm{mg} / \mathrm{kg}$ body weight). TA, extensor digitorum (EDL), and soleus (Sol) muscles were extracted and weighed. Left TA muscle samples were mounted on a piece of cork with OCT compound and frozen in isopentane cooled in liquid nitrogen for histological analysis. Samples were stored at $-80^{\circ} \mathrm{C}$ until use.

The TA muscle samples were cut into $10 \mu \mathrm{m}$ cross sections with a cryostat (CM3050S, Leica, Germany) at $-20^{\circ} \mathrm{C}$ and mounted on silanized slides for immunohistochemical and haematoxylin and eosin staining. After fixing with ice-cold $4 \%$ paraformaldehyde for $15 \mathrm{~min}$, sections were blocked at room temperature for $1 \mathrm{~h}$ with $10 \%$ normal goat serum (NGS) and $1 \%$ Triton X-100 in PBS and then washed twice in PBS for 5 min. Next, sections were incubated in 5\% NGS and $0.3 \%$ Triton X-100 in PBS for $16-20 \mathrm{~h}$ at $4^{\circ} \mathrm{C}$ with a primary antibody against dystrophin as a plasma membrane marker (1:500 dilution, Abcam, Tokyo, Japan), CD34 as a marker for endothelial cells ( $1: 250$ dilution, Abcam, Tokyo, Japan), and MMP-2 (1:2000 dilution, Abcam, Tokyo, Japan). The sections were washed several times with PBS, incubated with Alexa Fluor 488 or 568 conjugated secondary antibody $(1: 500$ dilution, Abcam, Tokyo, Japan) in 5\% NGS and 0.1\% Triton X$100 \mathrm{PBS}$ for $1 \mathrm{~h}$ at room temperature, and then mounted with Vectashield mounting medium. Images of TA muscle sections were obtained using a fluorescent light microscope (BX60; Olympus, Tokyo, Japan) and a CCD camera (DP72; Olympus, Tokyo, Japan). Digital images at 200x magnification were used to determine the cross-sectional area (CSA) of muscle fibers and the capillary number in each TA muscle. The FCSA of at least 75 fibers in each muscle were measured using Image-Pro Premier software (Media Cybernetics, Rockville, MD, USA). The total number of transverse capillaries and fibers in four random $154 \times 205 \mu \mathrm{m}$ fields was manually counted to determine the capillary density $(\mathrm{CD})$ and the $\mathrm{C} / \mathrm{F}$ ratio $[6,38]$. The MMP-2 positive areas were measured within the area of interest $(386 \times 386 \mu \mathrm{m})$ in each TA muscle.

Data are presented as mean \pm SD. Data sets were analyzed using one-way analysis of variance (ANOVA) followed by either the Bonferroni post hoc test or the Kruskal-Wallis test followed by Steel-Dwass multiple comparison tests, depending on the normality of the data distribution [39]. $P$ values less than 0.05 were considered significant.

\section{Results}

Body weight did not significantly differ between the groups (Table 1), though TA muscle weight significantly decreased relative to body weight after denervation $(P<0.05)$. Relative TA muscle weight was significantly higher in the DN + ES group compared to the DN group $(P<0.05)$. Relative EDL muscle weight was significantly lower $(P<0.05)$ in the DN and $\mathrm{DN}+\mathrm{ES}$ groups than in the control group, but no significant difference was observed between the DN and DN + ES groups (Table 1). Figure 1 shows representative images of TA muscle FCSA in the control, DN, and DN + ES groups. The CSA of TA muscle fibers were significantly smaller in the $\mathrm{DN}$ group than in the control and DN + ES groups $(P<0.05$, Table 1).

We examined the expression and localization of CD34, a capillary endothelial marker, and dystrophin, a sarcolemmal membrane marker (Figures 1(a)-1(c)). Immunohistochemical staining revealed that $\mathrm{CD} 34$-positive capillaries were observed around the dystrophin-positive myofibers. The C/F ratios in the control, DN, and DN + ES groups were $1.22 \pm$ $0.33,0.73 \pm 0.20$, and $1.17 \pm 0.26$, respectively. The $\mathrm{C} / \mathrm{F}$ ratio was significantly lower $(P<0.05)$ in the DN than in the control and DN + ES groups (Table 1). No significant differences were observed in capillary density between groups. MMP-2 was immunolocalized at the periphery of myofibers (Figure 2), and the MMP-2 positive area was significantly greater $(P<0.05)$ in the $\mathrm{DN}$ and the $\mathrm{DN}+\mathrm{ES}$ groups compared to the control group (Table 1).

\section{Discussion}

The current study presents new data on the effects of lowfrequency ES in early stages of disuse atrophy on denervated rat muscles. The results show that ES reduced the decrease in muscle weight, FCSA, and C/F ratio in early-stage DNinduced muscle disuse. The MMP-2 positive area increased in denervated TA muscle, whereas it remained unchanged in denervated TA muscle that was treated daily with ES.

Our results show that EDL and Sol muscle weight were lower in the DN and DN + ES groups than in the control group, and there was no difference between the DN and DN + ES groups. The EDL lies in a deeper layer than the TA, but 


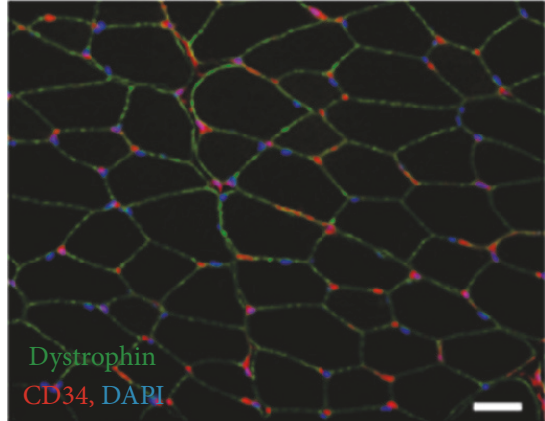

(a)

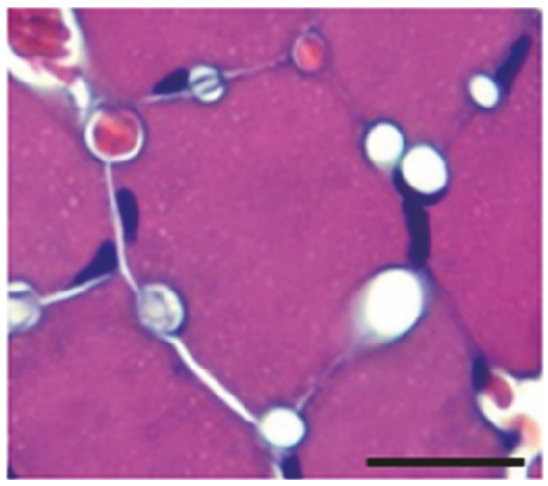

(d)

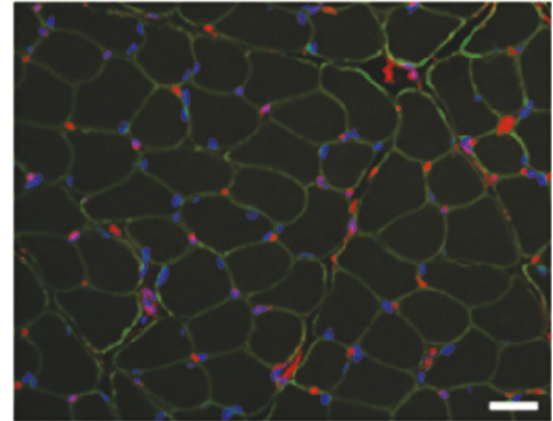

(b)

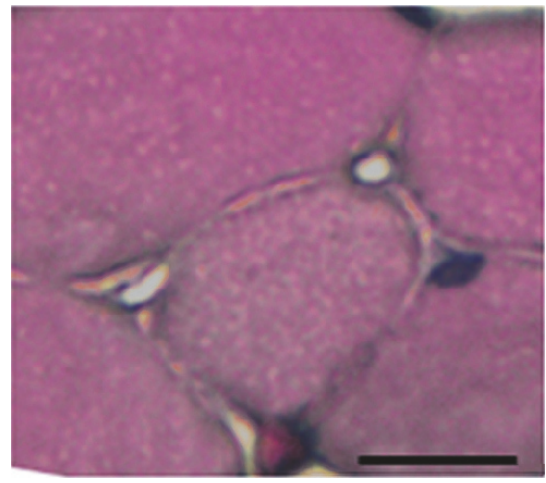

(e)

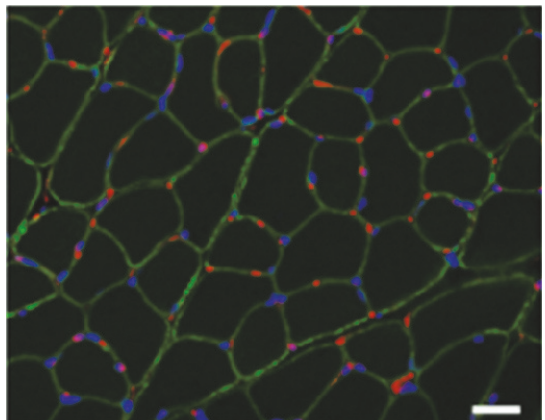

(c)

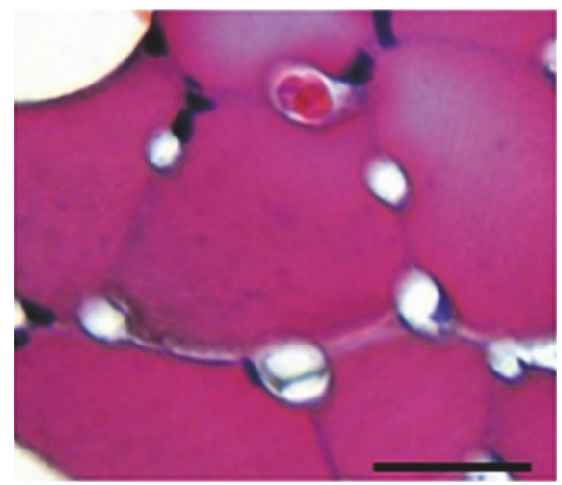

(f)

FIGURE 1: Immunohistochemical staining (a-c) for dystrophin (green), CD34 (red), and haematoxylin and eosin staining (d-f) in the CON $(a, e), D N(b, e)$, and DN + ES (c, f) groups in TA muscles. Scale bar $=25 \mu \mathrm{m}$.

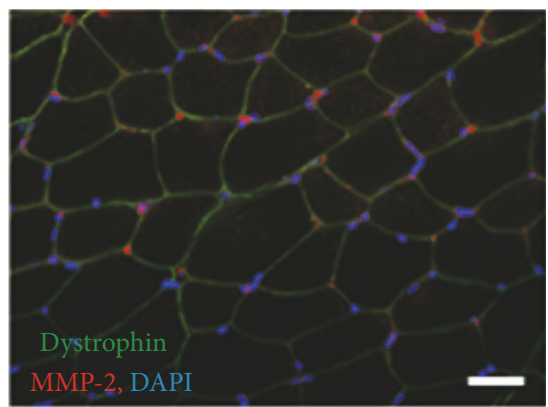

(a)

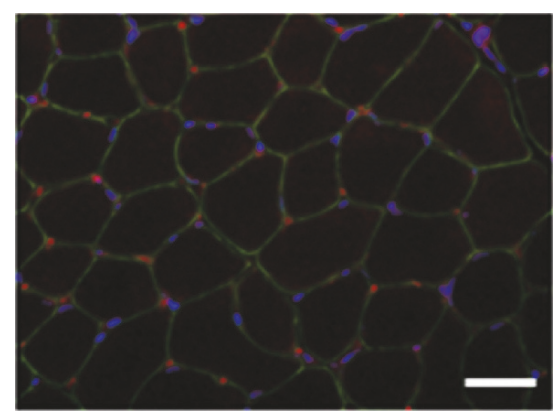

(b)

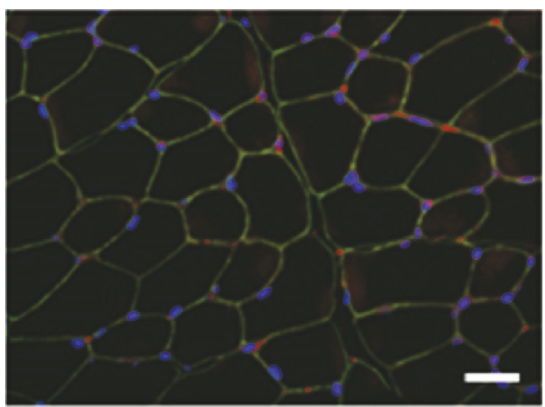

(c)

FIGURE 2: Immunohistochemical staining (a-c) for dystrophin (green), MMP-2 (red), and TA muscles in CON (a), DN (b), and DN + ES (c) groups. Scale bar $=25 \mu \mathrm{m}$.

both muscles are innervated by the tibial nerve, which is a branch of the sciatic nerve. Thus, the present data suggest that successful sciatic nerve denervation had no differential effect on the DN and the DN + ES groups. In general, relatively higher intensities or frequencies of ES are likely to result in greater effects on muscle atrophy and capillary regression in early-stage $\mathrm{DN}$-induced disuse. Muscle stimulation with higher-frequency ES regimens $(20-100 \mathrm{~Hz})$ tends to show beneficial effects of reducing the disuse-induced decreases in muscle mass, FCSA, and capillary supply [15, 25, 40-42]. However, a low-frequency ES regimen $(10 \mathrm{~Hz}, 8$ and $16 \mathrm{~mA}$,
$30 \mathrm{~min} /$ day, for 3 weeks) in stimulated TA muscle retarded the atrophy of denervated muscle [13]. Using the same ES conditions $(10 \mathrm{~Hz}, 16 \mathrm{~mA}, 30 \mathrm{~min} / \mathrm{day}$, for one week), the present study demonstrated that daily ES treatment reduced the DN-induced decrease in FCSA. Therefore, low-frequency ES treatment might have a beneficial effect on muscle disuse atrophy.

Direct ES has been reported to increase the $\mathrm{C} / \mathrm{F}$ ratio in intact fast-type dominant muscle $[42,43]$. Previous studies that compared the effects of different ES frequencies on resting blood flow and capillary density reported that 
optimal ES frequency for angiogenesis lies in the range of $10-40 \mathrm{~Hz}$ [44]. Moreover, previous reports describe a significant increase in $\mathrm{C} / \mathrm{F}$ ratio after only four to seven days of low-frequency ES $(10 \mathrm{~Hz})$ in intact TA and EDL muscles $[45,46]$. Our data showed that the $\mathrm{C} / \mathrm{F}$ ratios in the $\mathrm{DN}+\mathrm{ES}$ groups were significantly greater than in the DN group, but not different from the control group. This suggests that daily low-frequency ES treatment effectively ameliorates muscle capillary regression in early-stage $\mathrm{DN}$-induced disuse. However, our data also revealed that capillary density was unchanged following DN and ES treatment. In the present study, two-dimensional capillary density was determined by counting the total number of capillaries in a muscle section and was expressed as the number of capillaries per CSA unit. The measure of capillary density is likely subject to muscle atrophy or hypertrophy in fiber size if the $\mathrm{C} / \mathrm{F}$ ratio is stable. Hudlicka et al. suggested that muscle fiber hypertrophy is always associated with reduced capillary density [47]. However, there are discrepancies among previous reports; some found that muscle fiber atrophy is accompanied by increased capillary density two and eight weeks after denervation [5], whereas others report a decrease [48]. Nevertheless, longterm denervation results in reduced capillary density and $\mathrm{C} / \mathrm{F}$ ratio [49]. Some reports have also established that inactivity affects capillary density in slow and fast muscles differently $[5,50]$. Thus, the incongruent results regarding capillary density might be a result of differences in the duration of disuse, degree of muscle atrophy (fiber size), and muscle type.

A potential explanation for the effects of ES on capillary proliferation involves vascular endothelial growth factor (VEGF), which is known to be an important physiological regulator of angiogenesis. Several lines of evidence suggest that VEGF may also play a vasculoprotective role [51, 52]. In animal studies, ES-induced muscle contraction or mechanical stress facilitates cytokine (myokine) production [53] and increases blood flow and capillary vascularity $[54,55]$. Mechanical stress, such as shear stress and cyclic mechanical stretching, is known to induce VEGF expression $[56,57]$. In fact, continuous low-voltage ES in a hind limb ischemia model of rats for five days has been reported to increase VEGF production [58]. Therefore, the enhanced blood flow and cyclic stretching generated by ES-induced muscle contractions may stimulate VEGF production and lead to angiogenesis in the ES muscles.

Furthermore, MMPs play an important role in the homeostasis and maintenance of myofiber functional integrity and regulate skeletal muscle regeneration and angiogenesis by degrading ECM components [17, 18]. Capillary sprout formation is initiated by fragmentation of the capillary basal lamina and the subsequent migration and proliferation of distinct endothelial cell populations in response to angiogenic stimuli $[19,59]$. Various blood and connective tissue cells (e.g., macrophages, monocytes, and endothelial cells) secrete MMP-2 [60], and MMP-2 is involved in the degradation of type IV collagen, laminin, elastin, fibronectin, and many other ECM proteins [61]. Interestingly, an ablation study of MMP-2 reported downregulation of VEGF in the MMP2 knockout mouse [62], and MMP-2 ablation is known to impair the growth of regenerated muscle fibers and reduce angiogenesis via decreased VEGF, Flt-1, and nNOS expression. Thus, MMP-2 may influence angiogenesis and muscle regeneration via upregulation of VEGF and its receptor Flt1 and may also affect angiogenesis through regulation of nNOS-derived NO [21]. Our data revealed that the DN and $\mathrm{DN}+\mathrm{ES}$ groups had a greater MMP-2 positive area compared to the control group, but no difference was observed between the DN and DN + ES groups. Previous studies report increased MMP-2 activity following DN and limb immobilization $[24,25]$ and found that MMP-2 expression is elevated to different extents depending on the intensity of chronic ES [27, 28]. On the other hand, low-intensity ES $(20 \mathrm{~Hz}$ for $3 \mathrm{~s}$ followed by $6 \mathrm{~s}$ of rest, repeated for 20 maximal contractions, applied every $48 \mathrm{~h}$ for 28 days) did not alter MMP-2 activity [25]. Some discrepancies might exist among interventions with different conditions. A previous study reported that exercise-induced expression of MMP-2 is intensity dependent; high-intensity endurance exercise at approximately $70 \%$ of maximum oxygen consumption $\left(\mathrm{VO}_{2}\right.$ $\max$ ) increases MMP-2 expression, whereas low-intensity endurance exercise (approximately $50 \% \mathrm{VO}_{2} \max$ ) did not alter MMP-2 expression in skeletal muscles [27]. Although we did not determine $\mathrm{VO}_{2}$ during daily ES treatment, our ES regimen caused approximately $25 \%$ of maximal contraction force in the TA muscle [16]. Therefore, we may not have detected de novo alteration of MMP-2 in the DN + ES rats because of the low-intensity intervention.

The present findings should be interpreted in the context of their potential limitations. First, we used young, growing rats that were undergoing skeletal remodeling and thus needed the appropriate baseline controls. Although there was no inhibition of longitudinal bone growth of the tibiae in the young DN rats over time [63], young animals usually have greater potential for musculoskeletal recovery after limb disuse $[64,65]$. Our results might quantitatively differ if skeletally mature rats were used with a DN-induced disuse model. Second, our methodology precluded analysis of the activity of tissue inhibitors of metalloproteinases (TIMPs) and other pro- and antiangiogenic factors. A zymography analysis would be ideal to evaluate MMP-2 and TIMP1 activity, and analysis of inhibitors of metalloproteinases will be the focus of future experiments. Muscle atrophy is accompanied by changes in both MMPs and TIMPs, and the ratio between these metalloproteinases may be important in determining myoblast migration and differentiation $[61,66]$. Furthermore, MMPs and TIMPs play a significant role in regulating angiogenesis, which is the formation of new blood vessels [19]. For example, TIMP-2 plays an important role in ECM turnover by mediating the activity of MMPs, including MMP-2 [60]. Therefore, data on the balance between MMP-2 and TIMP-2 may provide useful information for evaluating the effects of low-frequency ES on reducing early-stage muscle atrophy and capillary regression in denervated muscles. Further studies using adult rats and other parameters and methodical techniques are needed for a more complete understanding of the relationship between ES-induced muscle contractions and capillary and muscle loss in the early stages of DN-induced muscle disuse. 
In conclusion, this is the first histomorphological study to our knowledge that assesses the influence of low-frequency ES on MMP-2 immunoreactivity, fiber atrophy, and capillary regression in denervated muscle in early stages of disuse. Our results showed that FCSA and C/F ratios decreased with increased MMP-2 immunoreactivity following DN and that daily low-frequency ES treatment on denervated muscle reduced the decrease in FCSA and C/F ratios but did not alter higher levels of MMP-2 produced by DN-induced disuse. These findings suggest that direct ES has beneficial effects on early-stage $\mathrm{DN}$-induced muscle disuse in the rat hind limb by ameliorating muscle atrophy and capillary regression without inducing a further increase in MMP-2 immunoreactivity. The use of direct ES to generate muscle contractions for the rehabilitation of skeletal muscles is well established, and the influence of different ES regimens on the balance of pro- and antiangiogenic factors is of great interest for future investigations as it may contribute to the understanding of the mechanisms responsible for the beneficial effects of ES.

\section{Conflicts of Interest}

The authors declare no conflicts of interest regarding the publication of this paper.

\section{Acknowledgments}

This study was supported in part by a Grant-in-Aid for Scientific Research (KAKENHI Grants nos. 25350829, 25282163, and 16K13021) and by a Grant-in-Aid for Developed Research from the Niigata University of Health and Welfare.

\section{References}

[1] S. C. Bodine-Fowler, S. Allsing, and M. J. Botte, "Time course of muscle atrophy and recovery following a phenol-induced nerve block," Muscle and Nerve, vol. 19, no. 4, pp. 497-504, 1996.

[2] M. Ishido, K. Kami, and M. Masuhara, "In vivo expression patterns of MyoD, p21, and Rb proteins in myonuclei and satellite cells of denervated rat skeletal muscle," American Journal of Physiology-Cell Physiology, vol. 287, no. 2, pp. C484-C493, 2004.

[3] R. R. Roy, H. Zhong, B. Siengthai, and V. Reggie Edgerton, "Activity-dependent influences are greater for fibers in rat medial gastrocnemius than tibialis anterior muscle," Muscle and Nerve, vol. 32, no. 4, pp. 473-482, 2005.

[4] H. Fujino, H. Kondo, F. Nagatomo, and A. Ishihara, "Capillary growth and regression in skeletal muscle," The Journal of Physical Fitness and Sports Medicine, vol. 3, no. 5, pp. 483-491, 2014.

[5] K. Tyml, O. Mathieu-Costello, L. Cheng, and E. G. Noble, "Differential microvascular response to disuse in rat hindlimb skeletal muscles," Journal of Applied Physiology, vol. 87, no. 4, pp. 1496-1505, 1999.

[6] A. Wagatsuma, H. Tamaki, and F. Ogita, "Capillary supply and gene expression of angiogenesis-related factors in murine skeletal muscle following denervation," Experimental Physiology, vol. 90, no. 3, pp. 403-409, 2005.
[7] U. Carraro, K. Rossini, W. Mayr, and H. Kern, "Muscle fiber regeneration in human permanent lower motoneuron denervation: relevance to safety and effectiveness of FES-training, which induces muscle recovery in SCI subjects," Artificial Organs, vol. 29, no. 3, pp. 187-191, 2005.

[8] H. Kern, S. Salmons, W. Mayr, K. Rossini, and U. Carraro, "Recovery of long-term denervated human muscles induced by electrical stimulation," Muscle and Nerve, vol. 31, no. 1, pp. 98101, 2005.

[9] M. Mödlin, C. Forstner, C. Hofer et al., "Electrical stimulation of denervated muscles: first results of a clinical study," Artificial Organs, vol. 29, no. 3, pp. 203-206, 2005.

[10] J. E. Stevens-Lapsley, J. E. Balter, P. Wolfe, D. G. Eckhoff, and W. M. Kohrt, "Early neuromuscular electrical stimulation to improve quadriceps muscle strength after total knee arthroplasty: a randomized controlled trial," Physical Therapy, vol. 92, no. 2, pp. 210-226, 2012.

[11] D. Gigo-Benato, T. L. Russo, S. Geuna, N. R. S. R. Domingues, T. F. Salvini, and N. A. Parizotto, "Electrical stimulation impairs early functional recovery and accentuates skeletal muscle atrophy after sciatic nerve crush injury in rats," Muscle and Nerve, vol. 41, no. 5, pp. 685-693, 2010.

[12] C. M. Pinheiro-Dardis and T. L. Russo, "Electrical stimulation based on chronaxie increases fibrosis and modulates TWEAK/Fn14, TGF- $\beta$ /myostatin, and MMP pathways in denervated muscles," American Journal of Physical Medicine \& Rehabilitation, vol. 96, no. 4, pp. 260-267, 2017.

[13] K. Tomori, Y. Ohta, T. Nishizawa, H. Tamaki, and H. Takekura, "Low-intensity electrical stimulation ameliorates disruption of transverse tubules and neuromuscular junctional architecture in denervated rat skeletal muscle fibers," Journal of Muscle Research and Cell Motility, vol. 31, no. 3, pp. 195-205, 2010.

[14] A.-C. D. Salter, F. J. R. Richmond, and G. E. Loeb, "Effects of muscle immobilization at different lengths on tetrodotoxininduced disuse atrophy," IEEE Transactions on Neural Systems and Rehabilitation Engineering, vol. 11, no. 3, pp. 209-217, 2003.

[15] N. Fujita, S. Murakami, T. Arakawa, A. Miki, and H. Fujino, "The combined effect of electrical stimulation and resistance isometric contraction on muscle atrophy in rat tibialis anterior muscle," Bosnian Journal of Basic Medical Sciences, vol. 11, no. 2, pp. 74-79, 2011.

[16] H. Tamaki, K. Tomori, K. Yotani et al., "Electrical stimulation of denervated rat skeletal muscle retards trabecular bone loss in early stages of disuse musculoskeletal atrophy," Journal of Musculoskeletal Neuronal Interactions, vol. 14, no. 2, pp. 220228, 2014.

[17] X. Chen and Y. Li, "Role of matrix metalloproteinases in skeletal muscle: migration, differentiation, regeneration and fibrosis," Cell Adhesion \& Migration, vol. 3, no. 4, pp. 337-341, 2009.

[18] S. A. Olenich, N. Gutierrez-Reed, G. N. Audet, and M. I. Olfert, "Temporal response of positive and negative regulators in response to acute and chronic exercise training in mice," Journal of Physiology, vol. 591, no. 20, pp. 5157-5169, 2013.

[19] Q. X. A. Sang, "Complex role of matrix metalloproteinases in angiogenesis," Cell Research, vol. 8, no. 3, pp. 171-177, 1998.

[20] H. W. Schnaper, D. S. Grant, W. G. Stetler-Stevenson et al., "Type IV collagenase(s) and TIMPs modulate endothelial cell morphogenesis in vitro," Journal of Cellular Physiology, vol. 156, no. 2, pp. 235-246, 1993.

[21] D. Miyazaki, A. Nakamura, K. Fukushima, K. Yoshida, S. Takeda, and S.-I. Ikeda, "Matrix metalloproteinase-2 ablation in 
dystrophin-deficient mdx muscles reduces angiogenesis resulting in impaired growth of regenerated muscle fibers," Human Molecular Genetics, vol. 20, no. 9, pp. 1787-1799, 2011.

[22] G. Lluri and D. M. Jaworski, "Regulation of TIMP-2, MT1-MMP, and MMP-2 expressioN during C2C12 differentiation," Muscle and Nerve, vol. 32, no. 4, pp. 492-499, 2005.

[23] M. Zimowska, E. Brzoska, M. Swierczynska, W. Streminska, and J. Moraczewski, "Distinct patterns of MMP-9 and MMP2 activity in slow and fast twitch skeletal muscle regeneration in vivo," International Journal of Developmental Biology, vol. 52, no. 2-3, pp. 307-314, 2008.

[24] A. Z. Reznick, O. Menashe, M. Bar-Shai, R. Coleman, and E. Carmeli, "Expression of matrix metalloproteinases, inhibitor, and acid phosphatase in muscles of immobilized hindlimbs of rats," Muscle and Nerve, vol. 27, no. 1, pp. 51-59, 2003.

[25] T. L. Russo, S. M. Peviani, J. L. Q. Durigan, and T. Fátima Salvini, "Electrical stimulation increases matrix metalloproteinase-2 gene expression but does not change its activity in denervated rat muscle," Muscle and Nerve, vol. 37, no. 5, pp. 593-600, 2008.

[26] S. M. Peviani, T. L. Russo, J. L. Q. Durigan et al., "Stretching and electrical stimulation regulate the metalloproteinase-2 in rat denervated skeletal muscle," Neurological Research, vol. 32, no. 8, pp. 891-896, 2010.

[27] E. Carmeli, M. Moas, S. Lennon, and S. K. Powers, "High intensity exercise increases expression of matrix metalloproteinases in fast skeletal muscle fibres," Experimental Physiology, vol. 90, no. 4, pp. 613-619, 2005.

[28] T. L. Haas, M. Milkiewicz, S. J. Davis et al., "Matrix metalloproteinase activity is required for activity-induced angiogenesis in rat skeletal muscle," American Journal of Physiology-Heart and Circulatory Physiology, vol. 279, no. 4, pp. H1540-H1547, 2000.

[29] J. Charan and N. D. Kantharia, "How to calculate sample size in animal studies?" Journal of Pharmacology and Pharmacotherapeutics, vol. 4, no. 4, pp. 303-306, 2013.

[30] G. Kerckhofs, M. Durand, R. Vangoitsenhoven et al., "Changes in bone macro- and microstructure in diabetic obese mice revealed by high resolution microfocus X-ray computed tomography," Scientific Reports, vol. 6, article 35517, 2016.

[31] H. Tamaki, K. Yotani, F. Ogita et al., "Electrical stimulation of denervated rat skeletal muscle ameliorates bone fragility and muscle loss in early-stage disuse musculoskeletal atrophy," Calcified Tissue International, vol. 100, no. 4, pp. 420-430, 2017.

[32] H. Sakakima, S. Kawamata, S. Kai, J. Ozawa, and N. Matsuura, "Effects of short-term denervation and subsequent reinnervation on motor endplates and the soleus muscle in the rat," Archives of Histology and Cytology, vol. 63, no. 5, pp. 495-506, 2000.

[33] H. Sakakima, Y. Yoshida, N. Morimoto, and K. Sakae, “The effect of denervation and subsequent reinnervation on the morphology of rat soleus muscles," Journal of Physical Therapy Science, vol. 14, no. 1, pp. 21-26, 2002.

[34] H. Takekura, H. Tamaki, T. Nishizawa, and N. Kasuga, "Plasticity of the transverse tubules following denervation and subsequent reinnervation in rat slow and fast muscle fibres," Journal of Muscle Research and Cell Motility, vol. 24, no. 7, pp. 439-451, 2003.

[35] H. Tamaki, K. Yotani, F. Ogita et al., "Changes over time in structural plasticity of trabecular bone in rat tibiae immobilized by reversible sciatic denervation," Journal of Musculoskeletal Neuronal Interactions, vol. 13, no. 3, pp. 251-258, 2013.

[36] H. Tamaki, K. Yotani, F. Ogita et al., "Effect of electrical stimulation-induced muscle force and streptomycin treatment on muscle and trabecular bone mass in early-stage disuse musculoskeletal atrophy," Journal of Musculoskeletal Neuronal Interactions, vol. 15, no. 3, pp. 270-278, 2015.

[37] K. Tomori, R. Kobayashi, T. Koseki, and Y. Ohta, "Effect of neuromuscular electrical stimulation of denervated muscle on the mRNA expression of IGFs in rat skeletal muscle and sciatic nerve," Journal of Physical Therapy Science, vol. 21, no. 3, pp. 269-273, 2009.

[38] F. N. Daussin, J. Zoll, S. P. Dufour et al., "Effect of interval versus continuous training on cardiorespiratory and mitochondrial functions: Relationship to aerobic performance improvements in sedentary subjects," American Journal of Physiology-Regulatory Integrative and Comparative Physiology, vol. 295, no. 1, pp. R264-R272, 2008.

[39] R. E. Andrews, K. M. Shah, J. M. Wilkinson, and A. Gartland, "Effects of cobalt and chromium ions at clinically equivalent concentrations after metal-on-metal hip replacement on human osteoblasts and osteoclasts: implications for skeletal health," Bone, vol. 49, no. 4, pp. 717-723, 2011.

[40] B.-T. Zhang, S. S. Yeung, Y. Liu et al., "The effects of low frequency electrical stimulation on satellite cell activity in rat skeletal muscle during hindlimb suspension," BMC Cell Biology, vol. 11, article 87, 2010.

[41] B.-S. Guo, K.-K. Cheung, S. S. Yeung, B.-T. Zhang, and E. W. Yeung, "Electrical stimulation influences satellite cell proliferation and apoptosis in unloading-induced muscle atrophy in mice," PLoS ONE, vol. 7, no. 1, Article ID e30348, 2012.

[42] S. Egginton and O. Hudlicka, "Selective long-term electrical stimulation of fast glycolytic fibres increases capillary supply but not oxidative enzyme activity in rat skeletal muscles," Experimental Physiology, vol. 85, no. 5, pp. 567-573, 2000.

[43] O. Hudlicka and S. Price, "The role of blood flow and/or muscle hypoxia in capillary growth in chronically stimulated fast muscles," Pflügers Archiv, vol. 417, no. 1, pp. 67-72, 1990.

[44] M. Shen, J. Gao, J. Li, and J. Su, "Effect of stimulation frequency on angiogenesis and gene expression in ischemic skeletal muscle of rabbit," Canadian Journal of Physiology and Pharmacology, vol. 87, no. 5, pp. 396-401, 2009.

[45] M. D. Brown, M. A. Cotter, O. Hudlická, and G. Vrbová, "The effects of different patterns of muscle activity on capillary density, mechanical properties and structure of slow and fast rabbit muscles," Pflügers Archiv, vol. 361, no. 3, pp. 241-250, 1976.

[46] F. M. Hansen-Smith, O. Hudlicka, and S. Egginton, "In vivo angiogenesis in adult rat skeletal muscle: early changes in capillary network architecture and ultrastructure," Cell and Tissue Research, vol. 286, no. 1, pp. 123-136, 1996.

[47] O. Hudlicka, L. Dodd, E. M. Renkin, and S. D. Gray, "Early changes in fiber profile and capillary density in long-term stimulated muscles," American Journal of Physiology-Heart and Circulatory Physiology, vol. 12, no. 4, pp. H528-H535, 1982.

[48] O. Mrázková and L. Puzanová, “The capillary bed in denervated muscle," Folia Morphologica, vol. 19, no. 1, pp. 71-81, 1971.

[49] E. I. Dedkov, T. Y. Kostrominova, A. B. Borisov, and B. M. Carlson, "Resistance vessel remodeling and reparative angiogenesis in the microcirculatory bed of long-term denervated skeletal muscles," Microvascular Research, vol. 63, no. 1, pp. 96-114, 2002.

[50] D. Desplanches, M. H. Mayet, B. Sempore, and R. Flandrois, "Structural and functional responses to prolonged hindlimb suspension in rat muscle," Journal of Applied Physiology, vol. 63, no. 2, pp. 558-563, 1987. 
[51] F. Kuwahara, H. Kai, K. Tokuda et al., "Hypoxia-inducible factor- $1 \alpha /$ vascular endothelial growth factor pathway for adventitial vasa vasorum formation in hypertensive rat aorta," Hypertension, vol. 39, no. 1, pp. 46-50, 2002.

[52] I. Zachary, "Signaling mechanisms mediating vascular protective actions of vascular endothelial growth factor," American Journal of Physiology-Cell Physiology, vol. 280, no. 6, pp. C1375-C1386, 2001.

[53] M. Scheler, M. Irmler, S. Lehr et al., "Cytokine response of primary human myotubes in an in vitro exercise model," American Journal of Physiology-Cell Physiology, vol. 305, no. 8, pp. C877-C886, 2013.

[54] S. Egginton and O. Hudlická, "Early changes in performance, blood flow and capillary fine structure in rat fast muscles induced by electrical stimulation," Journal of Physiology, vol. 515, no. 1, pp. 265-275, 1999.

[55] O. Mathieu-Costello, P. J. Agey, L. Wu, J. Hang, and T. H. Adair, "Capillary-to-fiber surface ratio in rat fast-twitch hindlimb muscles after chronic electrical stimulation," Journal of Applied Physiology, vol. 80, no. 3, pp. 904-909, 1996.

[56] N. G. dela Paz, T. E. Walshe, L. L. Leach, M. Saint-Geniez, and P. A. D'Amore, "Role of shear-stress-induced VEGF expression in endothelial cell survival," Journal of Cell Science, vol. 125, no. 4, pp. 831-843, 2012.

[57] T. P. Quinn, M. Schlueter, S. J. Soifer, and J. A. Gutierrez, "Cyclic mechanical stretch induces VEGF and FGF-2 expression in pulmonary vascular smooth muscle cells," American Journal of Physiology-Lung Cellular and Molecular Physiology, vol. 282, no. 5, pp. L897-L903, 2002.

[58] M. Nagasaka, M. Kohzuki, T. Fujii et al., "Effect of low-voltage electrical stimulation on angiogenic growth factors in ischaemic rat skeletal muscle," Clinical and Experimental Pharmacology and Physiology, vol. 33, no. 7, pp. 623-627, 2006.

[59] J. Folkman, "Angiogenesis in cancer, vascular, rheumatoid and other disease," Nature Medicine, vol. 1, no. 1, pp. 27-30, 1995.

[60] T.-S. Yu, Z. Li, R. Zhao, Y. Zhang, Z.-H. Zhang, and D.-W. Guan, "Time-dependent expression of MMP-2 and TIMP-2 after rats skeletal muscle contusion and their application to determine wound age," Journal of Forensic Sciences, vol. 61, no. 2, pp. 527533, 2016.

[61] E. Carmeli, M. Moas, A. Z. Reznick, and R. Coleman, "Matrix metalloproteinases and skeletal muscle: a brief review," Muscle and Nerve, vol. 29, no. 2, pp. 191-197, 2004.

[62] X. W. Cheng, M. Kuzuya, K. Nakamura et al., "Mechanisms underlying the impairment of ischemia-induced neovascularization in matrix metalloproteinase 2-deficient mice," Circulation Research, vol. 100, no. 6, pp. 904-913, 2007.

[63] H. Tamaki, K. Yotani, F. Ogita et al., "Changes over time in structural plasticity of trabecular bone in rat tibiae immobilized by reversible sciatic denervation," Journal of Musculoskeletal \& Neuronal Interactions, vol. 13, no. 3, pp. 289-296, 2013.

[64] T. Tanaka, Y. Kariya, and Y. Hoshino, "Histochemical study on the changes in muscle fibers in relation to the effects of aging on recovery from muscular atrophy caused by disuse in rats," Journal of Orthopaedic Science, vol. 9, no. 1, pp. 76-85, 2004.

[65] J. Tuukkanen, Z. Peng, and H. K. Vaananen, "The effect of training on the recovery from immobilization-induced bone loss in rats," Acta Physiologica Scandinavica, vol. 145, no. 4, pp. 407-411, 1992.

[66] M. P. Lewis, H. L. Tippett, A. C. M. Sinanan, M. J. Morgan, and N. P. Hunt, "Gelatinase-B (matrix metalloproteinase-9; MMP9) secretion is involved in the migratory phase of human and murine muscle cell cultures," Journal of Muscle Research and Cell Motility, vol. 21, no. 3, pp. 223-233, 2000. 


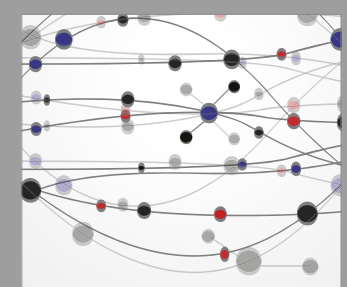

The Scientific World Journal
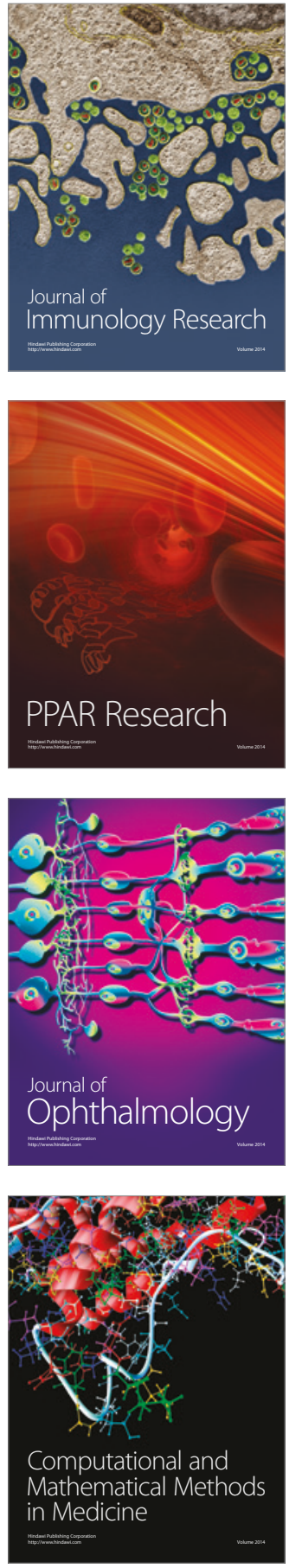

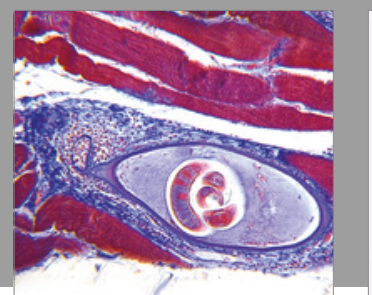

Gastroenterology Research and Practice
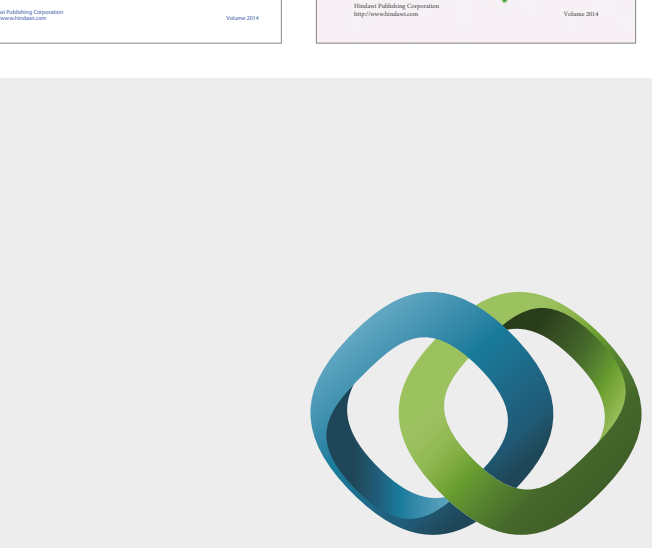

\section{Hindawi}

Submit your manuscripts at

https://www.hindawi.com
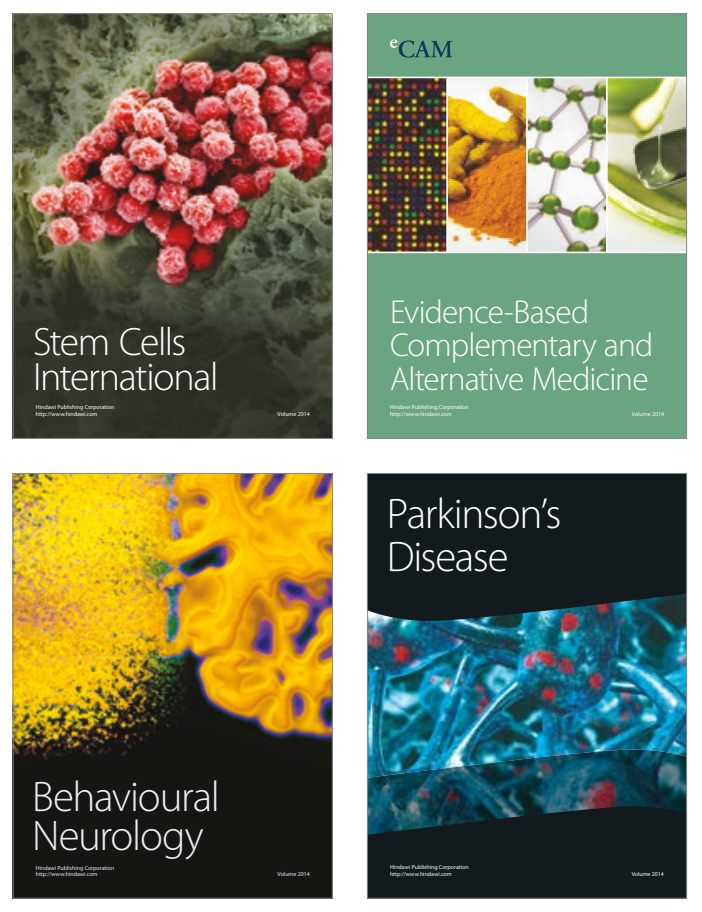
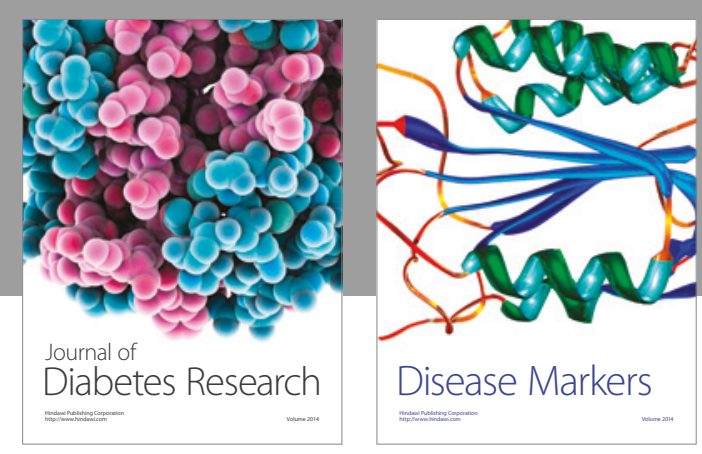

Disease Markers
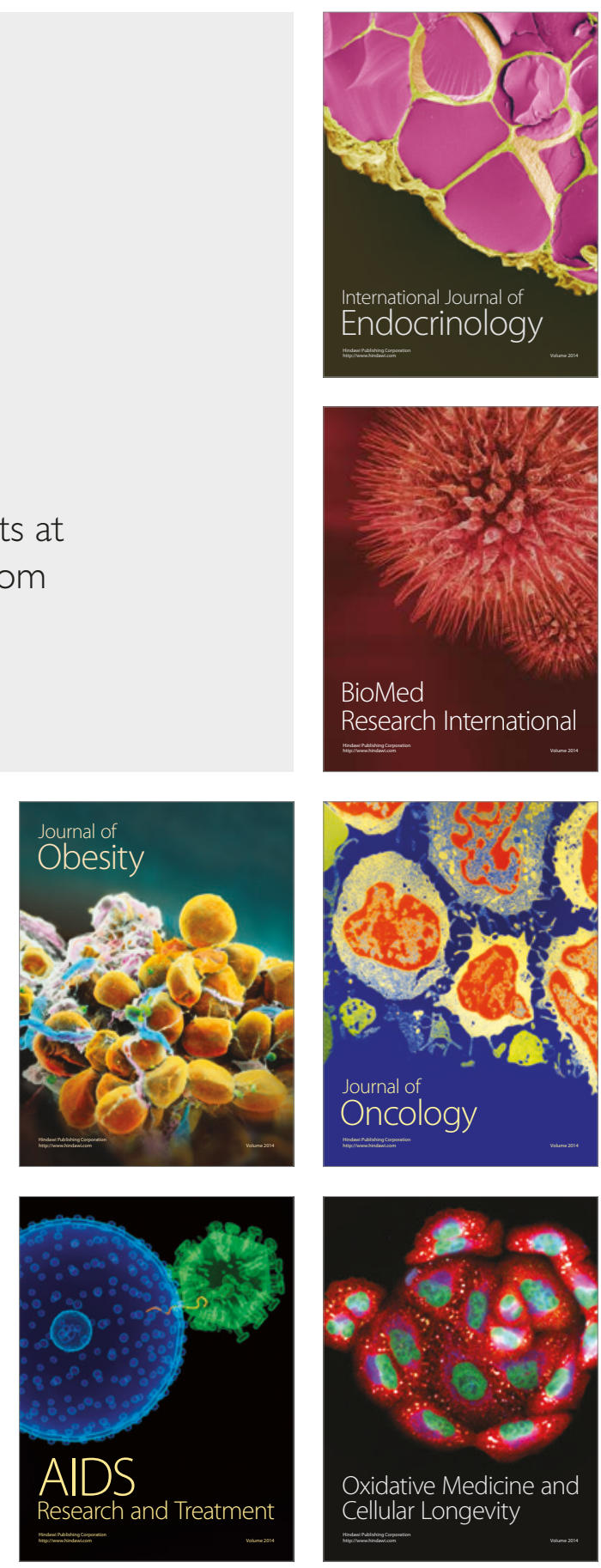